\title{
Vivência de acadêmicos de enfermagem frente à campanha de vacinação da covid-19 em populações ribeirinhas de um município do Amazonas
}

\author{
Experience of nursing students facing the covid-19 vaccination campaign in riverside \\ populations in a municipality in Amazonas
}
Experiencia de estudiantes de enfermería frente a la campaña de vacunación covid-19 en poblaciones ribereñas de un municipio de Amazonas

Marcelo Henrique da Silva Reis ${ }^{1 *}$, Jéssica Karoline Alves Portugal ${ }^{1}$, Karoline da Rocha Ferreira ${ }^{1}$, Dione Ferreira Oliveira ${ }^{1}$, Raynah Letícia Feitosa Torres ${ }^{1}$, Samara Souza Sodré ${ }^{1}$, Maria de Jesus de Menezes Rodrigues ${ }^{1}$, Dreiciane dos Santos Barbosa1, Yasmim de Souza Gomes', Yara da Silva dos Reis.

\section{RESUMO}

Objetivo: Descrever a experiência dos acadêmicos de enfermagem frente aos desafios na vacinação do COVID-19 nos ribeirinhos de um município do interior do Amazonas. Relato de experiência: Trata-se de um estudo do tipo descritivo, na modalidade relato de experiência que descreve as vivências de discentes de enfermagem de uma Universidade Federal situada no interior do Estado do Amazonas em parceria com a Secretaria Municipal de Saúde local (SEMSA), durante realização da campanha de vacinação contra Covid19. A parceria firmada entre as instituições visou garantir a vacinação de povos ribeirinhos de ambos os sexos, a partir de 18 anos, amparados por notas técnicas dos órgãos responsáveis do estado. Considerações finais: Participar dessa experiência inovadora para todos os acadêmicos vai somar na construção da carreira, principalmente por ajudar esses futuros profissionais a levarem o Sistema Único de Saúde (SUS) onde nenhum outro serviço de saúde chega, favorecendo o acesso universal aos povos ribeirinhos do interior do Amazonas. Mesmo com todas as adversidades, através da busca ativa e orientações realizadas, a equipe pôde contribuir para um maior alcance na cobertura vacinal nas comunidades ribeirinhas.

Palavras chave: Atenção primária à saúde, Covid-19, População rural.

\begin{abstract}
Objective: To describe the experience of nursing students facing the challenges of COVID-19 vaccination in riverside dwellers of a municipality in the interior of Amazonas. Experience report: This is a descriptive study, in the experience report modality, which describes the experiences of nursing students from a Federal University located in the interior of the State of Amazonas in partnership with the local Municipal Health Department (SEMSA), during the Covid-19 vaccination campaign. The partnership signed between the institutions aimed to guarantee the vaccination of riverside peoples of both sexes, from 18 years of age, supported by technical notes from the responsible state bodies. Final considerations: Participating in this innovative experience for all academics will add to the construction of a career, mainly by helping these future professionals to take the Unified Health System (SUS) where no other health service reaches, favoring universal access to peoples bordering the interior of the Amazon. Even with all the adversities, through the active search and guidance carried out, the team was able to contribute to a greater reach in vaccination coverage in the riverside communities.
\end{abstract}

Key words: Primary health care, Covid-19, Rural population.

1 Universidade Federal do Amazonas (UFAM), Coari - AM.

*E-mail: reis.henrique.marcelo@gmail.com

SUBMETIDO EM: 11/2021 


\section{RESUMEN}

Objetivo: Describir la experiencia de estudiantes de enfermería frente a los desafíos de la vacunación COVID 19 en pobladores ribereños de un municipio del interior de Amazonas. Informe de experiencia: Se trata de un estudio descriptivo, en la modalidad de informe de experiencia, que describe las experiencias de estudiantes de enfermería de una Universidad Federal ubicada en el interior del Estado de Amazonas en alianza con la Secretaría Municipal de Salud local (SEMSA), durante el Covid -19 campaña de vacunación. La alianza suscrita entre las instituciones tuvo como objetivo garantizar la vacunación de los pueblos ribereños de ambos sexos, a partir de los 18 años, respaldada por notas técnicas de los órganos estatales responsables. Consideraciones finales: Participar de esta experiencia innovadora para todos los académicos se sumará a la construcción de una carrera, principalmente al ayudar a estos futuros profesionales a llevar el Sistema Único de Salud (SUS) donde ningún otro servicio de salud llega, favoreciendo el acceso universal a los pueblos limítrof es del interior. del Amazonas. Aún con todas las adversidades, a través de la búsqueda activa y la orientación realizada, el equipo pudo contribuir a un mayor alcance en las coberturas de vacunación en las comunidades ribereñas.

Palabras clave: Atención primaria de salud, Covid-19, Población rural.

\section{INTRODUÇÃO}

O mundo passou por um grande impacto entre o final de 2019 e início de 2020. Foi descoberto na China um vírus com grande potencial de contágio e que em pouco tempo teve alcance a todos os continentes do globo. A Organização Mundial de Saúde (OMS) reconheceu como pandemia a contaminação pelo novo coronavírus, causador da doença Covid-19 (SARS-CoV-2), que trouxe medo, apreensão e muitas perdas em um curto intervalo. Em pouco tempo, o vírus foi capaz de mudar a rotina do mundo todo, se tornando uma emergência de preocupação internacional (PORTUGAL JKA, 2020; SENHORAS EM, 2020; THE LANCET, 2020; WORLD HEALTH ORGANIZATION (WHO), 2020).

Com o passar dos primeiros meses, a doença infectou e levou a óbito milhões de pessoas ao redor do mundo, mesmo com todas as medidas de restrições implementadas por grande parte dos governos das maiores nações, visto que se tratava de algo totalmente desconhecido, que estava vitimando pessoas de todas as classes sociais, mesmo as em estado de saúde considerado ótimo. Em âmbito nacional, o Ministério da Saúde já contabilizou mais de 20 milhões de casos, destes, quase 600.000 brasileiros perderam suas vidas para o vírus. O estado do Amazonas foi um dos primeiros do país a declarar estado de calamidade pública, pois o crescimento no número de casos estava incontrolável, pessoas estavam morrendo por falta de leitos adequados e oxigênio. Todo esse reflexo estadual fez com que o vírus chegasse nas regiões mais longínquas do território local, principalmente nas comunidades ribeirinhas que estão isoladas geograficamente das sedes municipais (CASTRO FF, et al., 2020; SENHORAS EM, 2020).

Em meio as estratégias adotadas para combater o impacto e propagação do novo vírus, iniciou-se uma verdadeira corrida para a formulação de uma vacina eficaz para as diversas variantes que pelo mundo se disseminaram. Após longos e acelerados estudos oriundos de diversas farmacêuticas, em dezembro de 2020 foi aprovada a primeira vacina no mundo, desenvolvida pela parceria entre as farmacêuticas Pfizer e BioNTech. As vacinas são produtos termolábeis, podendo haver alteração ou transformação se expostos a temperaturas variáveis, neste sentido, a Amazônia Brasileira possui elevadas temperaturas, razão pela qual é indispensável mantê-las preservadas em condições favoráveis de conservação para serem administradas na população de forma segura e eficaz (DOMINGUES CMAS, 2021).

$\mathrm{Na}$ vacinação não há estratégia exclusiva, no entanto, utiliza-se aquela que assegura a obtenção e a manutenção de um alto índice de cobertura vacinal. A Vacinação extramuros, que são atividades realizadas fora das unidades de saúde, domicílio a domicílio, especialmente as pessoas que vivem em áreas rurais, de difícil acesso, possibilitando alcançar populações que de outra maneira, certamente nunca seriam vacinadas. Como resultado, a imunização extramuros conseguiu alcançar as regiões remotas como as comunidades ribeirinhas do Amazonas, formadas por moradores que possuem relação íntima com a natureza, retirando dela grande parte do seu sustento (SANTOS EP, 2017). 
Na região amazônica existem diversas peculiaridades, que comparadas a outras regiões do país, podem refletir significativamente nas condições de vida e saúde da população. No município que ocorreram as atividades, essa realidade não é diferente, as comunidades ribeirinhas são o espaço onde eles costumam estreitar seu convívio social. Dentre as dificuldades para o acesso à essas comunidades, podemos citar as longas distâncias, dificuldades de transporte, alto custo de deslocamento, precárias condições de saneamento, a falta de profissionais da saúde e a dificuldade de comunicação (DIEGUES AC, et al., 2000; GAMA ASM, 2016; GAMA ASM, et al., 2018; SOUSA IS, 2009).

Neste sentido, o presente relato teve como objetivo descrever a experiência dos acadêmicos de enfermagem frente aos desafios na vacinação do COVID-19 nos ribeirinhos de um município do interior do Amazonas.

\section{RELATO DE EXPERIÊNCIA}

Trata-se de um estudo do tipo descritivo, na modalidade relato de experiência que descreve as vivências de discentes de enfermagem de uma Universidade Federal situada no interior do Estado do Amazonas em parceria com a Secretaria Municipal de Saúde local (SEMSA), durante realização da campanha de vacinação contra Covid-19. A parceria firmada entre as instituições visou garantir a vacinação de povos ribeirinhos de ambos os sexos, a partir de 18 anos, amparados por notas técnicas dos órgãos responsáveis do estado.

O roteiro de cada viagem foi planejado pela equipe de saúde da SEMSA, considerando a distância entre as comunidades que são subdivididas em calhas de rios e lagos do Alto Solimões, Médio Solimões, Baixo Solimões e Lago do Mamiá. A distância geralmente dessas comunidades até a sede municipal varia entre 1 a 4 horas de viagem a depender da potência da embarcação. As atividades de imunização foram realizadas durante 03 dias do mês de junho de 2021, iniciando às 06:30 da manhã e retornando às 19:00 horas. Todo custeio das viagens foi amparado pela Prefeitura, desde a compra de insumos básicos como água e alimentação, aluguel de embarcação, combustível e disponibilização de profissionais de nível superior para contribuir e supervisionar a equipe em área.

O transporte até as comunidades ribeirinhas se deu por meio de uma lancha rápida coberta, com capacidade para 11 pessoas e motor potência 90HP (Horse Power - força em cavalos). O rio nesse período estava na época de uma grande enchente, dificultando o acesso as casas ribeirinhas, um dos obstáculos para a busca ativa. O café da manhã era realizado dentro da lancha, durante o trajeto até o primeiro destino, estratégia adotada para ganhar tempo. O material para preparo do almoço era entregue à um Agente Comunitário de Saúde (ACS) de alguma comunidade que receberia vacinação, para que fosse feito por alguma liderança local quando a equipe retornasse à comunidade.

Ao chegar nas comunidades, primeiramente era acionado o ACS, para que então ele pudesse guiar a equipe e montar a base de apoio. Em seguida, a equipe se dividia em duas: uma equipe saía em busca ativa para informar e identificar moradores aptos para a vacinação e os documentos necessários para preenchimento da ficha de notificação, enquanto a outra equipe organizava os materiais necessários e o local que serviria de ponto de vacinação (geralmente um centro comunitário, escola, igreja ou residência) (JUNIOR JCFP, et al., 2020).

O local da vacinação era dividido em recepção (local em que eram conf eridos os documentos necessários, registrado no cartão de vacina os dados da vacina como nome, lote e responsável pela aplicação), local de preparo e aplicação da vacina. Antes da aplicação da vacina, o ribeirinho era orientado individualmente quanto ao mecanismo de ação da vacina, possíveis reações adversas e condutas para minimização das mesmas, além de ser alertado quanto a importância da dose de reforço. Nesse momento, era nítido o temor de alguns adultos e idosos à vacina ou aos ef eitos adversos, mostrando a importância de se conhecer e repassar informações pertinentes à vacina para evitar traumas e negação.

Foram visitadas 18 comunidades ribeirinhas nas calhas em que a equipe realizou intervenção, vacinando em total de 155 pessoas de 18 anos ou mais, incluindo a primeira e segunda dose das vacinas AstraZeneca e Coronavac. Os colaboradores para esta ação foram sete acadêmicas do $8^{\circ}$ semestre da graduação em enfermagem, uma enfermeira da Unidade Básica de Saúde Fluvial, que esteve vacinando e também supervisionando a viagem, além do preceptor da aula prática da Universidade e o motorista da lancha. 
A busca ativa obteve mais retorno no Médio Solimões em relação ao Baixo Solimões, o que sugere que quanto mais distante a comunidade, menor é o acesso à informação e aos serviços de saúde. As comunidades do Médio Solimões estavam com acesso mais fácil, considerando que a maioria ainda possuía terra firme. A adesão nessas comunidades foi muito maior e a busca ativa muito mais eficaz. Longas caminhadas foram realizadas nessas comunidades, pois a distância entre uma casa e outra é relativamente grande, algumas vezes ultrapassando 10 minutos para ir e retornar até a base, nas áreas alagadas dessa região só era possível realizar busca ativa por meio de canoa e remo.

As comunidades do Baixo Solimões visitadas estavam completamente alagadas, dificultando a comunicação com os moradores. O transporte dentro dessas comunidades se dava por bote disponibilizado pelo ACS, visto que devido a correnteza do rio, estava impossibilitado o uso da canoa a remo. Essas comunidades possuíam postes de energia elétrica, que se encontravam entre 70 e $90 \%$ submersos, of erecendo um risco de choque elétrico para os moradores. Foi relatado pela população desses locais que apagões de energia são comuns e chegam a durar mais de três semanas devido ao difícil acesso, e que nesse período de alagações é redobrado os cuidados principalmente com crianças, pois os fios da rede elétrica ficam ao alcance de todos.

As mulheres representaram o sexo mais prevalente, com $52 \%$, em relação a faixa etária, os indivíduos de 18 a 59 anos corresponderam à $96 \%$ dos vacinados nos três dias de campanha, do total geral, $2 \%$ eram profissionais da saúde, $4 \%$ eram profissionais da educação da zona rural, $2,5 \%$ eram gestantes ou puérperas e $91,5 \%$ eram ribeirinhos (agricultores, pescadores e donas de casa).

Foi ressaltado para a população vacinada a necessidade de completar o esquema vacinal com a dose de reforço, visto que não haverá vacinação extramuros para a segunda dose. Portanto, os vacinados eram orientados a procurar na data marcada para dose de reforço a Unidade Básica de Saúde Ribeirinha ou qualquer outra unidade básica de saúde.

\section{DISCUSSÃO}

O período da cheia (que favorece a of erta de peixes e consequentemente a pesca) atraia muitos ribeirinhos (majoritariamente homens) para a prática pesqueira e posterior venda na zona urbana, razão que impossibilitou a vacinação das pessoas que haviam saído para pescar ou realizar outras atividades floresta adentro. Também é possível observar que os homens sempre são minorias quando informamos sobre a ida aos serviços de saúde. Esta informação pode ter relação com o fato de culturalmente serem a maioria dos gestores financeiros de suas residências, trabalhando fora para trazer sustento, ficando fora de casa grande parte do tempo e consequentemente estando menos nos serviços de saúde do que as mulheres, que por outros lado são consideradas as responsáveis por cuidar da saúde de suas famílias, além disto, elas possuem características na fisiologia que as difere dos homens, fato que pode levar a uma maior procura de cuidar da saúde (GAMA ASM, et al., 2018; GUIMARÃES AF, et al., 2020; REIS MHS, et al., 2020).

A vacinação mostra-se como o principal meio de controle da propagação do vírus Sars-Cov-2 em meio ao atual cenário sanitário mundial, aliada às medidas de prevenção e higiene estabelecidas. O planejamento para vacinação conforme oferta de imunizantes ao Brasil é uma difícil tarefa, considerando as diversas características climáticas e geológicas do território nacional. De maneira singular, a Amazônia pela vasta riqueza em rios e florestas, necessita de fortes investimentos para uma boa cobertura vacinal (PORTUGAL JKA, et al., 2021).

A zona rural do município representa para a equipe de estratégia da saúde uma inquietação, visto que a distância das comunidades à sede do município gera dificuldades tanto no acesso dos ribeirinhos aos serviços de saúde quanto das equipes de saúde no que tange ao deslocamento para esses locais. O principal meio de locomoção no Amazonas é o transporte hidroviário através de barcos, lanchas e canoas à remo, algo propício para o agravamento de casos infectados por COVID, devido à distância para acesso e resistência aos tratamentos of erecidos nas Unidades de Cuidados Intensivos (UCl) (FRANCO EC, et al., 2015; FRAXE TJP, et al., 2007; GUIMARÃES AF, 2020). 
A distância de uma comunidade para outra e a distribuição das casas é irregular. Para acessar algumas casas nessas localidades são necessárias longas caminhadas em terra firme ou locomoção através de canoas a remo. O papel de um ACS é indispensável para a busca ativa, pois o território na maioria das vezes é completamente desconhecido pela equipe de saúde. Este profissional tem grande importância para a saúde, principalmente em áreas rurais, onde ele possui atribuições peculiares a região que reside, além de todas as atribuições impostas pela Política Nacional de Atenção Básica, que em sua última atualização pode agregar funções mais complexas, porém, trará grandes benefícios se realizadas de forma correta (MINISTÉRIO DA SAÚDE, 2017; REIS MHS, et al., 2021).

A recusa de aplicação da vacina da Covid-19 é agravada por diversos fatores, dentre os principais observados podemos citar: opção religiosa, medo das reações adversas, preocupação com o intervalo para consumo de bebida alcóolica, além de acreditarem em correntes de redes sociais que definem a vacina como algo maléfico e que pode ocasionar a morte ou complicações à saúde. A baixa escolaridade facilita a disseminação de falsas informações, mas ao mesmo tempo favorece o convencimento após uma boa apresentação sobre o processo e os resultados positivos que a vacinação em massa pode trazer (REIS MHS, et al., 2021).

Apesar de a vacinação representar o principal meio de erradicação de doenças infecciosas, a falta de políticas públicas que visem a conscientização quanto a importância da vacinação interferem diretamente na adesão. Isso foi confirmado quando conferido os cartões de vacinas, onde foi possível observar que a maioria estavam incompletos, alguns apresentando anos de atraso, mesmo com esses indivíduos tendo cobertura da Unidade Básica de Saúde Fluvial pelo menos uma vez a cada 4 meses (REIS MHS, et al., 2020).

A capacitação da equipe envolvida na vacinação é a chave para uma boa cobertura vacinal, pois é necessário envolver o público, explicar sobre o processo de fabricação da vacina, informar sobre condutas para amenizar possíveis reações, alertar sobre a importância da vacinação em massa para a comunidade e enfatizar a necessidade da dose de reforço. Os argumentos científicos somam para uma boa aceitação, destacando aí a importância dos profissionais em conhecer as particularidades do público alvo para que o repasse dessas informações pertinentes seja feito de maneira compreensível (MILLER DG, et al., 2020).

Participar dessa experiência inovadora para todos os acadêmicos vai somar na construção da carreira, principalmente por ajudar esses futuros profissionais a levarem o Sistema Único de Saúde (SUS) onde nenhum outro serviço de saúde chega, favorecendo o acesso universal aos povos ribeirinhos do interior do Amazonas. Também ficou evidente o fato de estar frente a frente com a realidade de recusa das vacinas, são situações que se contadas em entrevistas ou em roda de conversas podem muito bem serem taxadas como fantasiosas, mas constatar isso in loco mostra que a falta de informação e o conhecimento que a ciência tem para a medicina moderna pode ceifar vidas. Mesmo com todas as adversidades, através da busca ativa e orientações realizadas, a equipe pôde contribuir para um maior alcance na cobertura vacinal nas comunidades ribeirinhas.

\section{REFERÊNCIAS}

1. CASTRO FF, et al. Idosos ribeirinhos da Amazônia Brasileira no enfrentamento da covid -19. $2^{\mathrm{a}}$ ed. Brasília: Editora ABEn, 2020; 142-149p.

2. DIEGUES AC, etal. Os Saberes Tradicionais e a Biodiversidade no Brasil. $1^{\text {a }}$ ed. São Paulo:Probio-MMA, 2000;211p.

3. DOMINGUES CMAS. Desafios para a realização da campanha de vacinação contra a Covid -19 no Brasil. Cadernos de Saúde Pública (CSP), 2021;37(1): e00344620.

4. FRANCO EC, et al. Promoção da saúde da população ribeirinha da região amazônica: relato de experiência. Rev CEFAC, 2015.

5. FRAXE TJP, et al. Comunidades ribeirinhas amazônicas: modos de vida e uso dos recursos naturais. Manaus: EDUA; 2007.

6. GAMA ASM, et al. Inquérito de saúde em comunidades ribeirinhas do Amazonas, Brasil. Cad. Saúde Pública, 2018; 34(2): e00002817.

7. GAMA ASM. Automedicação em comunidades ribeirinhas na região do Médio Solimões, Amazonas. Tese (Doutorado em Enfermagem na Saúde do Adulto). Universidade de São Paulo (USP), São Paulo, 2016; 115 p. 
8. GUIMARÃES AF, et al. Acesso a serviços de saúde por ribeirinhos de um município no interior do estado do Amazonas, Brasil. Rev Pan Amaz Saude, 2020;11: e202000178.

9. JúNIOR JCFP, et al. Atuação de acadêmicos de enfermagem nas ações de enfrentamento da covid-19 em comunidades ribeirinhas do amazonas: relato de experiência. Revista eletrônica Acervo Saúde, 2020; $12(10):$ e5049.

10. MILLER DG, et al. The role of medical students during the COVID-19 pandemic. Ann Intern Med. 2020; 173(2): 145146.

11. MINISTÉRIO DA SAÚDE. Portaria no 2.436, de 21 de setembro de 2017. Aprova a Política Nacional de Atenção Básica, estabelecendo a revisão de diretrizes para a organização da Atenção Básica, no âmbito do Sistema Único de Saúde (SUS). 2017. Disponível em: https://bvsms.saude.gov.br/bvs/saudelegis/gm/2017. Acessado em: 20 de novembro de 2021.

12. PORTUGAL JKA, et al. Acadêmicos de enfermagem do interior do amazonas em missões ribeirinhas durante a pandemia de covid-19. Revista eletrônica Acervo Saúde, 2020;3(11): e9320.

13. PORTUGAL JKA, et al. Percepção do impacto emocional da equipe de enfermagem diante da pandemia de COVID 19: relato de experiência. Revista eletrônica Acervo Saúde, 2020;46(Esp.): e3794.

14. REIS MHS, et al. Características da população ribeirinha de um município do interior do Amazonas. Revista eletrônica Acervo Saúde, 2021;13(11): e9273.

15. REIS MHS, et al. O impacto do advento de uma Unidade Básica de Saúde Fluvial na assistência aos povos ribeirinhos do Amazonas. Revista eletrônica Acervo Saúde, 2020;53(Sup.): e3631.

16. SANTOS EP. Guia de boas práticas de Imunização em áreas remotas de difícil acesso. Sociedade Brasil eira de Imunização (SBIm), 2017,33p.

17. SENHORAS EM. Novo Coronavírus e seus impactos econômicos no mundo. Boletim de Conjuntura (BOCA), 2020; 1(2): 39-42.

18. SOUSA IS. As condições de vida e saneamento nas comunidades da área de influência do gasoduto Coari -Manaus em Manacapuru - AM. Hygeia. 2009; 5(9): 88-98.

19. THE LANCET. COVID-19: protecting health-care workers. Lancet, 2020.

20. WORLD HEALTH ORGANIZATION (WHO). 2020. Doença por coronavírus 2019 (COVID -19): relatório de situação 36. Disponível em: https://www.who.int/docs/default-source/coronaviruse/situation-reports/20200225. Acessado em: 20 de novembro de 2021. 